\title{
Neural cytoskeleton capabilities for learning and memory
}

\author{
Avner Priel • Jack A. Tuszynski • Nancy J. Woolf
}

Received: 29 November 2008 / Accepted: 6 April 2009 /

Published online: 15 May 2009

(C) The Author(s) 2009. This article is published with open access at Springerlink.com

\begin{abstract}
This paper proposes a physical model involving the key structures within the neural cytoskeleton as major players in molecular-level processing of information required for learning and memory storage. In particular, actin filaments and microtubules are macromolecules having highly charged surfaces that enable them to conduct electric signals. The biophysical properties of these filaments relevant to the conduction of ionic current include a condensation of counterions on the filament surface and a nonlinear complex physical structure conducive to the generation of modulated waves. Cytoskeletal filaments are often directly connected with both ionotropic and metabotropic types of membraneembedded receptors, thereby linking synaptic inputs to intracellular functions. Possible roles for cable-like, conductive filaments in neurons include intracellular information processing, regulating developmental plasticity, and mediating transport. The cytoskeletal proteins form a complex network capable of emergent information processing, and they stand to intervene between inputs to and outputs from neurons. In this manner, the cytoskeletal matrix is proposed to work with neuronal membrane and its intrinsic components (e.g., ion channels, scaffolding proteins, and adaptor proteins), especially at sites of synaptic contacts and spines. An information processing model based on cytoskeletal networks is proposed that may underlie certain types of learning and memory.
\end{abstract}

Keywords Cytoskeleton • Actin • Microtubules • Memory $\cdot$ Learning • Information processing

A. Priel · J. A. Tuszynski $(\bowtie)$

Department of Physics, University of Alberta, Edmonton, AB, T6G 2J1, Canada

e-mail: jtus@phys.ualberta.ca

J. A. Tuszynski

Division of Experimental Oncology, Cross Cancer Institute,

11560 University Avenue, Edmonton, AB, T6G 1Z2, Canada

N. J. Woolf

Behavioral Neuroscience, Department of Psychology, University of California, Los Angeles, CA 90095-1563, USA 


\section{Introduction}

Ample empirical evidence supports the premise that learning and memory involve reorganization of the neuronal cytoskeleton [1-3]. Although learning and memory are often considered together, learning is the process by which the nervous system improves its adaptation to the environment, whereas memory represents information stored in neurons or in the connections between them. While biological memory may operate somewhat similarly to content-addressable computer memory $[4,5]$, it is notably more robust and is capable of retrieval based on incomplete inputs, whereas logical memory is by far more accurate and demanding.

The memory trace is envisioned as a physical substrate-something substantive and concrete. As such, a viable neural correlate for memory is the reorganization of the cytoskeleton, especially a restructuring of those microtubules and actin filaments responsible for connecting structures in the cell body to synapses or axon terminals. By changing these intra-neuronal cytoskeletal connections, synaptic strength might increase or decrease, the distribution of inputs might become skewed to favor activation of certain dendrites over others, or the release of neurotransmitters might be modified. A most exciting possibility is that the neuronal cytoskeleton possesses the capability to permanently encode information at a subcellular level. Such a proposal is consistent with several lines of experimental evidence.

Several lines of empirical evidence strongly support the proposal that microtubules are reorganized with learning and memory, including both correlative and interventive measures. In one correlative study, researchers found that threefold increases in microtubules paralleled passive avoidance training [6]. In another correlative study, cDNA microarrays revealed up-regulation of the microtubule-associated protein-2 (MAP2) gene, with excess levels of the protein associating with $\beta$-tubulin in trained chicks as compared to controls, indicative of cytoskeletal remodeling with learning [3]. Using an interventive approach, the microtubule toxin, colchicine, impaired performance on several learning paradigms, which included the Morris water maze, radial arm maze, aversive conditioning, and an operant conditioning task [7-10]. As colchicine is well known to block the depolymerization of microtubules, these multiple studies indicate that learning relies on dynamic microtubules, participating in polymerization and depolymerization cycles and interacting with other microtubules as well as with actin filaments.

There are other gene expression and protein binding studies indicating that additional cytoskeletal proteins are necessary for converting short-term memory into long-term memory, a process termed "memory consolidation" $[11,12]$. During memory consolidation, actin, tubulin, and F-actin capping proteins increase their binding to other proteins [12]. Cytoskeletal protein dynamics are additionally modulated by MAPs and growth-associated proteins, such as GAP-43, stathmin, and SCG10 [13]. Memory consolidation is also accompanied by MAP2 expression alterations and stathmin binding increases [11, 12].

In this paper, we intend to present the building blocks of a model that addresses the issue of the molecular substrate of memory and learning within the structure of the neuronal cytoskeleton. We will pay particular attention to the role and properties of actin filaments and microtubules in this regard. Although other subcellular organelles could similarly participate, the cytoskeletal matrix is unique in the manner in which it interconnects various synaptic sites. 


\section{Learning and memory: cytoskeletal plasticity in dendrites and spines}

Across diverse learning paradigms, the dendrite-specific binding protein MAP2 is repeatedly implicated, suggesting that plasticity in dendrites and spines is most critical to learning and memory. Social isolation results in recognition memory deficits and is also correlated with decreased levels of $\alpha$-tubulin and MAP2 in the hippocampus [14]. Fear conditioning to tone or to the training chamber context produces marked changes in MAP2 immunohistochemical staining in the auditory cortex or hippocampus, respectively [15-17]. These staining alterations are interpreted as evidence that MAP2 is proteolyzed with tone or contextual learning, which is consistent with Western blot analyses revealing an increase in breakdown products having a molecular weight of $90 \mathrm{kDa}$. Proteolysis of MAP2 occurs throughout the cell body and apical dendrites of the $\sim 15 \%$ of cortical and hippocampal neurons containing elevated levels of MAP2, many of which are large pyramidal cells [18]. Similar learning-related changes in MAP2 have been shown to parallel avoidance training and be accompanied by altered staining patterns for muscarinic receptor and protein kinase $\mathrm{C}$ (PKC) [19].

In addition to MAP2 being altered by learning, conditions that impair learning and memory also disrupt MAP2. Cerebral hypoperfusion, which impairs performance on the Morris water maze, also correlates with decreased MAP2, GAP-43, and synaptophysin [20]. The senescence-accelerated (SAMP10) mouse strain that exhibits learning and memory deficits also expresses less cortical MAP2 and has cortical neurons with fewer apical dendrites [21]. MAP2 is likely to be involved in memory consolidation as a stabilizer of the cytoskeleton. A plausible explanation as to why MAP2 is broken down with memory consolidation is that the existing microtubule matrix is broken down before the new matrix can be built. Rather than merely being a structural matrix, the MAP2-micotubule matrix also tethers together signal transduction molecules. To illustrate, transgenic mice having the N-terminus of MAP2 truncated are markedly impaired on a contextual learning task and also demonstrate deficits in binding cAMP-dependent kinase (PKA), which leads to a reduced capacity to phosphorylate MAP2 [22].

In addition to MAP2, the smaller MAP tau is involved in learning and memory, as has been shown in transgenic studies. Adding excess of MAP tau by way of a transgene resulted in a measurable impairment in olfactory learning in Drosophila and spatial reference memory in mice $[23,24]$. Importantly, increased tau in P301 transgenic mice is correlated with improved memory before tau has a chance to aggregate, whereas memory is impaired after tau has aggregated [25]. One hypothesis is that excess tau overly increases the stability of microtubules and that this is a deterrent to normal microtubule dynamics ordinarily at play in learning [23]. Accordingly, normal learning function requires a fine balance between stability and instability in microtubules.

Changes in the ratio of dynamic to stable microtubules have been noted in laboratory animals exposed to restraint stress, which, like memory, involves cytoskeletal remodeling [26]. Following exposure to restraint stress, mice demonstrated significantly decreased levels of tyrosinated tubulins and significantly increased levels of acetylated tubulins in the hippocampus, indicative of a shift away from a more dynamic cytoskeleton. Thus, the intrinsic lattice/mosaic structure of microtubules is altered. This would be a convenient mechanism for memory - at least short-term memory. Given that synaptic machinery turns over in a matter of hours to days, the notion of a more or less permanent site for memory 
in the cytoskeleton is very appealing. In this regard, the periodic structure of microtubules based on the distribution of tyrosinated and acetylated tubulins offers the possibility for information representation essential to learning and memory. Changes have also been noted in the dynamics of neurofilaments attendant with synaptic remodeling associated with depression or treatments with antidepressants [27, 28].

Spines, which are located on select dendrites, undergo more rapid and extensive reorganization than do dendrites. Spine plasticity depends on actin filaments and is a likely correlate of learning. Recently, it has been shown that extinction to contextual fear (i.e., a reduction of an existing fear response) depends on actin filament rearrangements in spines [29]. Actin-rich spines also show distinct types of morphological change that correlate with long-term potentiation (LTP), an experimentally induced enhanced response recorded from hippocampal tissue slices and observed in behaving animals [30]. LTP has an initial, intermediate, and late phase, and it is during the intermediate phase that increases have been noted in local concentrations of mRNA for MAP2 and $\mathrm{Ca}^{2+}$-calmodulin-dependent kinase II (CaMK II) [31]. CaMK II is a critical learning-related kinase that phosphorylates MAP2 and AMPA receptor subunits, steps that are fundamental to the potentiated synaptic response [32]. Increased MAP1B phosphorylation, for example, occurs within minutes of the induction of LTP [33].

\section{Electric signaling along the cytoskeletal matrix and memory}

Until recently, little has been known about electric signaling in microtubules and actin filaments. Thus, it is only beginning to be possible to address the issue of how the cytoskeletal matrix might participate in information processing during learning and memory. Learningrelated adaptations in cytoskeletal proteins - microtubules, actin filaments, and their related proteins (such as the MAP and Arp families) - have mostly been interpreted as functional equivalents to cytoskeletal changes with neural plasticity during development where new structure is the major result. The most popular theory in neurobiology is that memory is represented by altered connections or modified synaptic strengths among large assemblies of interconnected neurons [34]. This proposal dates back to Canadian psychologist Donald Hebb and his description of the now classic "Hebbian synapse" in which synaptic knobs continuously extend and retract in much the manner spines are now known to behave.

These hypothesized Hebbian changes in synaptic strength have been verified in studies employing the LTP paradigm. Potentiated synapses demonstrated by the LTP method last anywhere from minutes to weeks or longer [35]. LTP expression is typically near maximal following each isolated learning experience; however, the range shifts upwards with subsequent learning experiences [36]. Since this shift cannot be expected to continue to rise indefinitely, a permanent representation of the memory trace, which could be stored in the microtubule-actin filament matrix, would allow synaptic physiology to return to baseline [37]. Signaling within the cytoskeletal matrix would be expected to be particularly critical to information storage that lasts longer than LTP is able to persist, since eventual LTP decay seems likely.

Although alterations in the cytoskeletal matrix might be considered as already being incorporated in the Hebbian model, we consider the intraneuronal matrix model of neural computing as having important distinctions. The cytoskeletal matrix model directly incorporates an intrinsic mechanism accounting for self-organization and autonomy insofar 
as the cytoskeleton regulates its own reorganization. Experimental evidence obtained from behaving animals indicates that synaptic potentiation frequently reaches a saturation point or ceiling and that conversion to structural synaptic reorganization may provide longer term storage [38]. Storing information in the intraneuronal matrix (as compared to storing information as potentiated synapses or as altered connections) provides a way for synapses to return to baseline conditions without loss of information. This proposal is supported by reports of potentiated synapses returning to baseline within days after learning $[39,40]$ and other findings that the dendritic structure of neurons becomes more and more elaborate with learning and experience [41]. The notion that the adaptations within the intraneuronal matrix, rather than (or in addition to) changes to interneuronal connectivity, are involved with learning and memory is consistent with species-specific patterns of connective plasticity. There is a more limited potential for restructuring of cortical circuits among primates compared to rodents [42], whereas human and non-human primates clearly have more advanced cognitive aptitudes than rodents. The potential for cytoskeletal matrix reorganization is implied by the fine structure of neurons. Human pyramidal neurons have more extensive dendrite arbors and spine densities compared to mouse pyramidal neurons [43] and accordingly possess more extensive intraneuronal cytoskeletal matrices.

Information storage in the microtubule-actin filament matrix requires that co-incident inputs and spatiotemporal patterns of synaptic input have some means to be encoded. Some forms of LTP depend on the glutamate $N$-methyl-D-aspartic acid (NMDA) receptor. NMDA receptor-dependent LTP has been proposed as a model for a neural coincidence detector because the postsynaptic cell must be depolarized before the NMDA receptor will permit flow of $\mathrm{Ca}^{2+}$ across the membrane [44]. LTP also obeys the spatiotemporal learning rule insofar as neurons show heightened sensitivity to repeated stimulus intervals and to inputs that are synchronous but spatially distinct $[45,46]$.

Electric signaling by actin filaments and microtubules has the potential to participate in coincidence detection and storage of spatiotemporal patterns of inputs to a much greater extent than potentiation at individual synapse because these cytoskeletal matrices have the potential to interconnect any of the hundreds of thousands of synapses within an individual neuron in as many different configurations as are mathematically possible. Synaptic inputs to the cell activate receptors that in turn interact with scaffolding proteins, adaptor proteins, and actin filaments concentrated in the spines; these actin filaments in turn interact with microtubules in the dendrites, the cell body, and the axon. Because actin filaments associate with the membrane throughout the neuron and microtubules are found everywhere except inside the cell nucleus, synaptic inputs to sites virtually anywhere on the neuron can be transmitted to virtually any other site. Electric signals transmitted by way of actin filaments to actin-microtubule cross-linker proteins to microtubules and by way of MAPs and signal transduction molecules to other microtubules represent a potential intraneuronal system of wiring capable of conducting ionic waves. In the next section, we briefly outline how actin filaments may conduct ionic waves within the neuron.

\section{Electric signal propagation by actin filaments}

Actin filaments form a highly suitable conduit for electric signal propagation because they are highly concentrated just below the surface of the neuronal membrane, and they 
are extensively interconnected with microtubules and neurofilaments found throughout dendrites, axons, and cell bodies [47]. Moreover, several specialized structures, such as spines and growth cones, are enriched with actin filaments. Actin filaments typically extend from the neuronal membrane deep into the sub-spine region [48]. Spines and growth cones possess highly dynamic actin filaments essential to their roles in neural growth and development, experience-related plasticity, and reorganization with increased or decreased neural stimulation $[49,50]$. The structural matrix formed by actin filaments is decorated with many functional proteins. Actin filaments work with binding and adaptor proteins to anchor or tether a variety of signal transduction molecules, receptors, and ion channels [51].

Among the mainly structural roles attributed to actin filaments, none have taken into account actin filaments being polyelectrolytes with charged groups that interact with counterions in the surrounding media. It is because actin filaments are polyelectrolytes surrounded by counterions that they possess the capacity of transmitting signals or sustaining ionic conductances $[52,53]$. The conductance of ionic waves enables actin filaments to act like "electric cables." Biophysical properties such as the electric dipoles of actin monomers, counterion condensation of actin filaments, and the linear charge density along the longitudinal axis of the actin filament underlie actin filaments being capable of ionic wave conduction.

The actin monomer is a globular protein containing four sub-domains, as determined by the resolved crystal structure of actin bound to ADP to a resolution of $1.54 \AA$ [54]. Whether ADP or ATP is bound to the actin monomer affects its polymerization into filaments and its association to actin-binding proteins. ADP versus ATP binding also results in different three-dimensional conformations for the actin monomer; these conformational shifts mainly occur at the active binding site and the sensor loop [55].

Actin filaments, being rod-like polymers, are particularly likely to have counterions adsorbed to their surface, since the Onsager-Manning-Oosawa condensation principle predicts that any polyelectrolyte having a charge density over 1.0 electron per Bjerrum length, $\lambda_{\mathrm{B}}$, will have counterions adsorbed to its surface [56]. At low ionic concentrations, ions disperse in the solution surrounding actin filaments; however, at high ionic concentrations, such as those in the intraneuronal environment, ions would be expected to densely adsorb to the surface of actin filaments due to complementary charges. Counterions that are adsorbed to the surface of actin filaments are critical to the ionic wave conductances along these filaments (Fig. 1).

Cantiello et al. [52] discovered that actin filaments generated electrical signals. Their first experiment evaluating actin filament responses to osmotic pressure demonstrated that actin filaments possess a conductive capability, responding to electric fields in the range of $500-2,000 \mathrm{~V} / \mathrm{cm}$. From their experiments, they were able to determine that actin filament conductance was dependent on the counterions that adsorbed to the surface of the filament. The electrical conductivity they measured also depended on $\mathrm{pH}$ and was abolished at $\mathrm{pH}$ 5.5. These researchers devised a more elaborate experimental setup to more completely study this electrical phenomenon in actin filaments [58].

Actin filaments have a linear charge density of $1.65 \times 10^{2} \mathrm{e} / \mathrm{nm}$, which allows them to carry electric charge in the form of ionic flow along the longitudinal axis of the filament [53]. As illustrated in Fig. 2, because of shielding, ions are able to travel along actin filaments without significantly affected by the surrounding environment [53]. Actin filaments also possess the capacity to store excess charge, with the capacitance per monomer being $\sim 96 \times 10^{-6} \mathrm{pF}$. The velocity of electrical signals along actin filaments is calculated to be in the range of $\sim 1-100 \mathrm{~m} / \mathrm{s}$, i.e., in the approximate range of neural impulse transmittance. 


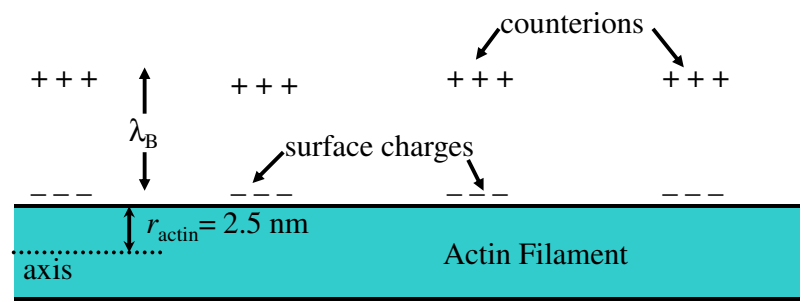

Fig. 1 Counterion charge density illustration. Under conditions of dense ionic concentrations, multivalent linear waves of counterions condense on the surface of actin filaments. Additional counterions congregate around the filament approximately one Bjerrum length away from the surface. At $T=293 \mathrm{~K} \lambda_{\mathrm{B}}$ is typically $\sim 7.13 \times 10^{-10} \mathrm{~m}$. Adapted from [57]

Thus, it is conceivable that simultaneous propagation of ionic current occurs along actin filaments and the axonal membrane.

There is additional evidence that actin filaments respond to electric and magnetic fields. Electrical current applied to actin filaments suspended in a solution-filled well located between two gold electrodes results in those actin filaments aligning parallel to the electric lines and bridging the intervening gap between electrodes [59]. Other studies similarly indicate that actin filaments possess the ability to align parallel and perpendicular to the electric field depending on the nature of the field $[60,61]$. It is also conceivable that electric or magnetic fields contribute to neural structure. Meggs [62] hypothesized that electric fields could be responsible for the structural organization of actin filaments and microtubules, with dipole moments intrinsic to actin and tubulin aligning these polyelectrolytes parallel to the main direction of the electric field.

\section{Microtubules propagate and amplify electrical signals}

In the living cell, most microtubules are composed of 13 protofilaments, which have alternating $\alpha$ - and $\beta$-tubulin monomers as shown in Fig. 3. The lattice structure of the microtubule is such that each tubulin monomer is bound to six other tubulins, with the presence or absence of a seam specifying the A or B lattice type [63, 64]. The bonds between tubulin also contribute to the specific biophysical properties of microtubules, and the

Fig. 2 Actin filaments support a traveling ionic cloud which is affected by the dipole moments of monomers (based on [53])

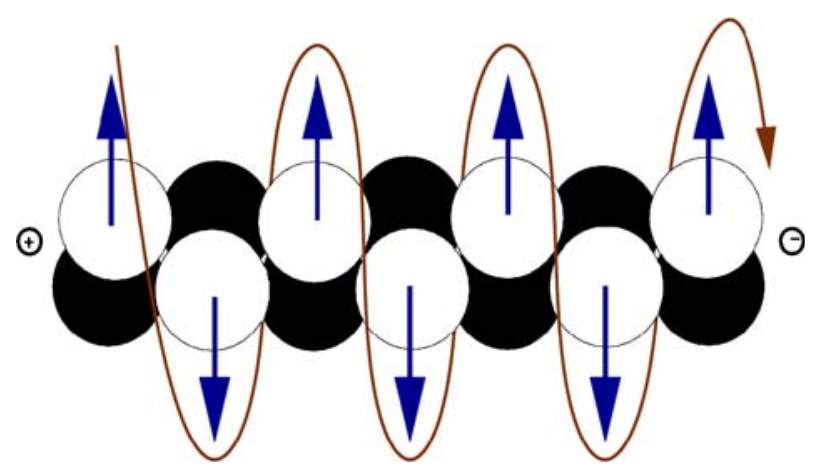



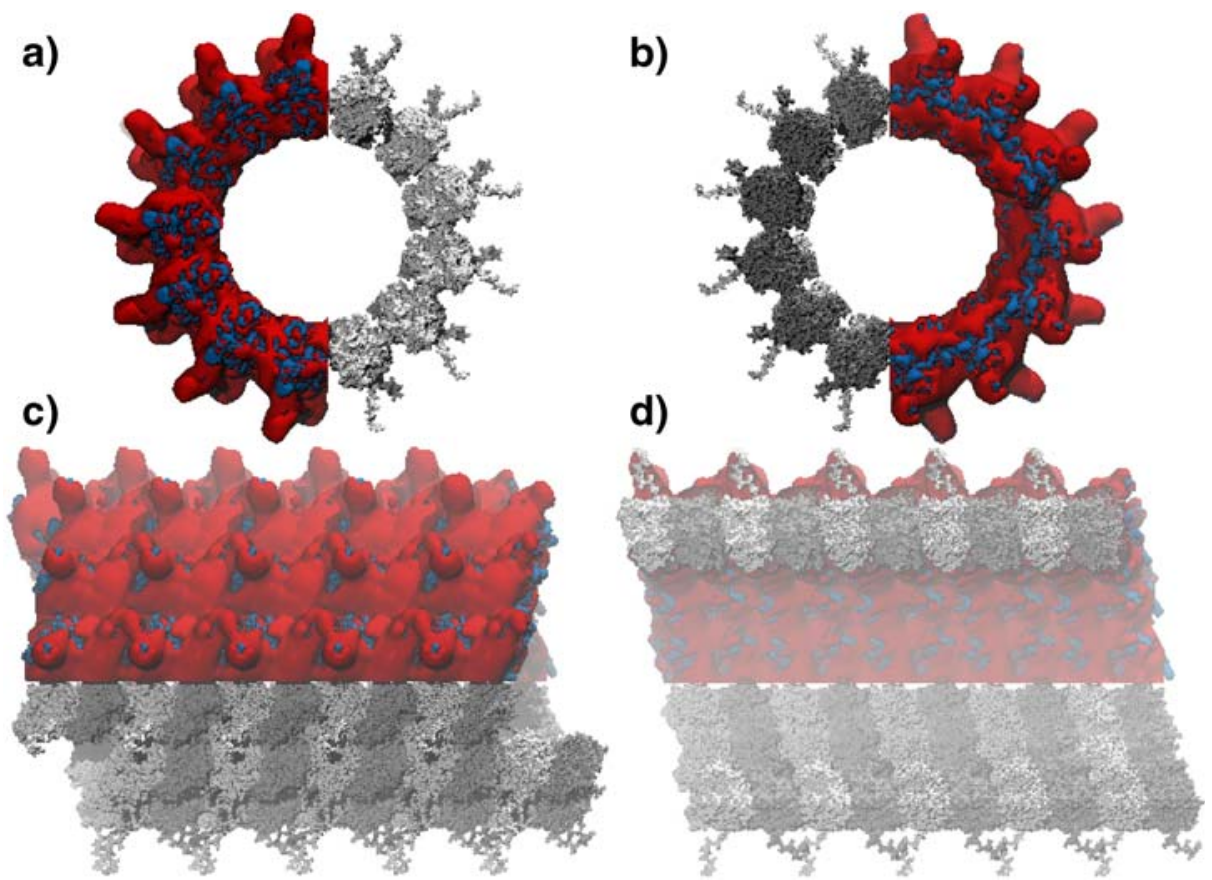

Fig. 3 The structure of a microtubule as shown from the plus end (a), the minus end (b), the outside (c), and the inside (d). This figure was produced by Dr. T. Luchko with the help and assistance of Dr. Tianshen Zhou and Mr. Paul Greidanus at Center of Excellence in Integrated Nanotools (CEIN) of the University of Alberta

assembly of a microtubule follows different patterns depending on the availability of cations and other features of the surrounding environment [65]. Cryo-electron microscopic analyses of microtubules indicate that the inter-dimer interface is responsible for the tendency of $\alpha$-tubulin to adopt a straight conformation and $\beta$-tubulin to adopt a curved conformation [66]. The curved versus straight conformation of a microtubule is partly attributable to GTP hydrolysis (to GDP), which only occurs for $\beta$-tubulin. Additionally, the strength of lateral bonds will affect whether microtubule protofilaments remain relatively straight or curved. Since tubulins are staggered across adjacent protofilaments, lateral contacts can, under most conditions, contribute greatly to stability. It is the curved conformation that favors microtubule disassembly. Longitudinal bonds between tubulins also contribute to stability and are stronger than lateral bonds between protofilaments by $\sim 7 \mathrm{kcal} / \mathrm{mol}[63,64]$.

The mostly electronegative outer surface of microtubules is particularly concentrated on the ridges of the protofilaments [67]. Many positive surfaces lie buried in the underlying regions between microtubule protofilaments. This longitudinal arrangement of charge along microtubules contributes to a sizable linear charge density. Minoura and Muto [68] found the linear charge density along microtubule protofilaments to be $2.5 \mathrm{e} / \mathrm{nm}$.

Ferroelectricity (i.e., the ability to spontaneously generate dipole moments) is another enigmatic feature of microtubules. Assembled microtubules are ferroelectric insofar as they exhibit spontaneous dipole moments, the directions of which can be controlled by external electric fields [69-72]. This ferroelectric capability contributes to the conductive properties 
of microtubules and can serve as a biophysical basis for modeling signaling in microtubules. There are three different ways that dipoles can be arranged in a microtubule lattice. The arrangements can be random, strongly ferroelectric or parallel, or weakly ferroelectric [69]. The weakly ferroelectric arrangement leads to dipole-dipole interactions at the couplings of tubulin to its six nearest neighbors that can be described as being in conflict or "frustrated." Conflicting or frustrated dipole-dipole interactions produce "kink-like" excitations that propagate down the microtubule [69]. These kink-like excitations have also been termed "solitary waves," defined as traveling solitons or defects. As the kink-like excitation passes along the microtubule, a switch in the dipole moment of the tubulin monomer occurs.

There is also an elastic coupling of the traveling wave and the energy of GTP hydrolysis calculated at $6.25 \times 10^{-20} \mathrm{~J}$ [70]. The kink-like excitation or traveling solitary wave partly relies on this elastic coupling, and as a result, the solitary wave carries the free portion of this energy of GTP hydrolysis. This is in agreement with a pseudo-spin model of GTP hydrolysis that similarly suggests GTP hydrolysis is a critical factor in determining the dipole state of the tubulin dimer [71]. Also, there is a double-well potential in the tubulin dimer that can be attributed to a mobile electron, which is localized either to the $\alpha$ - or $\beta$-tubulin dimer $[71,72]$. Viewed in the pseudo-spin model, a state change of this mobile electron would be coupled to GTP hydrolysis. However, a different model proposes that dipole "flip waves" travel along microtubules caused by tubulins alternating between GTP and GDP states, which can occur without actual changes in GTP binding [73].

Electrical signaling along microtubules has been studied using both experimental and theoretical approaches. Direct experimental observations were accomplished using a dual patch-clamp setup. Using this setup, taxol-stabilized microtubules behaved much like biomolecular transistors, responding to brief pulses of electric current ranging $\pm 200 \mathrm{mV}$ [74]. As shown in Fig. 4, isolated microtubules not only conducted but also amplified applied electrical current twofold. This observed conductivity of ionic waves along microtubules appeared to depend on the condensed positive counterion cloud distributed along the length of the microtubule ( $20 e^{-}$per tubulin monomer). An ionic cloud is found above the electronegative surface charge of the microtubule. Calculations based on experimental data are consistent with the conclusion that microtubules are capable of nonlinear wave propagation and behave like biological transistors [74].

Microtubules have also been shown capable of conductance using an electro-orientation approach [68]. For intact microtubules, conductance is $157 \pm 7 \mathrm{mS} / \mathrm{m}$, and for microtubules treated with subtilisin, $96 \pm 6 \mathrm{mS} / \mathrm{m}$. Given that subtilisin removes C-termini from microtubules, it appears likely that counterions on the surface, and particularly those on the negatively charged C-termini, are responsible for the observed conductance.

The electrical signaling of microtubules has also been approached from a theoretical point of view, being modeled as a nonlinear electrical circuit [75]. The derivative calculations of this biophysical model are consistent with the experimental observations for ionic conduction along microtubules and the amplitude amplification of propagated ionic flow. The model mimics the behavior of a microtubule in solution in which the microtubule cylinder's core is separated from the rest of the ions in the bulk solution by the counterion condensation cloud. This cloud acts as a dielectric medium between the two, providing resistive and capacitive components for the behavior of the dimers that make up the microtubule. Ion flow is predicted at a radial distance from the center of the cylinder, which is approximately equal to the Bjerrum length. There is an inductive component to the electrical properties of ionic waves due to the helical nature of the microtubule structure, much like a solenoid induces a helical ion flow. 
Fig. 4 Electric current is amplified by a microtubule as shown in [74]. a Electrostatic distribution of counterions inside and outside the microtubule are responsible for propagation of the electric signal. b Electric current is applied to one end of the microtubule and collected at the other end, showing a remarkable $\mathrm{I}-\mathrm{V}$ characteristic (adapted from [74])

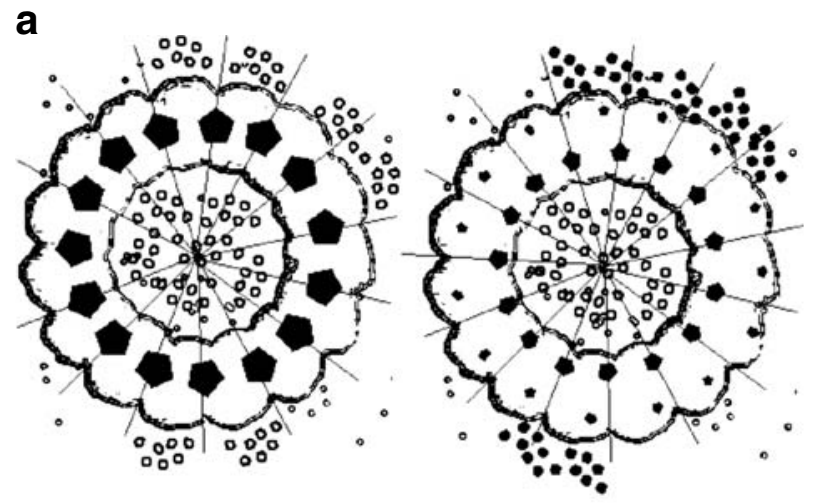

b

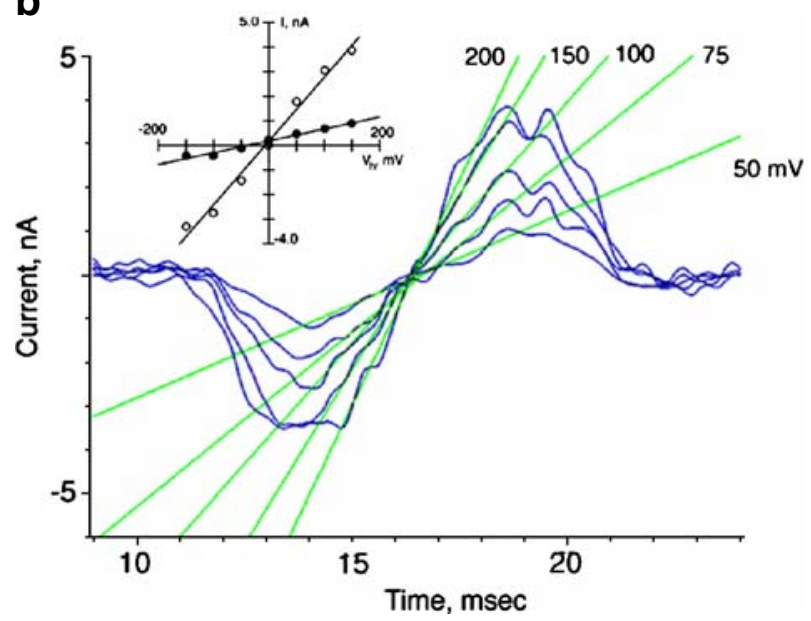

As reported in [75], the model equation requires a discrete potential being introduced in one section of the microtubule where Kirchhoff's laws for the currents and voltages are applied. After taking the discrete equations to the continuum limit, an equation for the potential $V$ for small spatial and temporal variations is obtained:

$$
\begin{aligned}
L C_{0} \frac{\partial^{2} V}{\partial t^{2}}= & a^{2} \partial_{x x} V+R_{2} C_{0} \frac{\partial}{\partial t}\left(a^{2} \partial_{x x} V\right)-R_{1} C_{0} \frac{\partial V}{\partial t}+2 R_{1} C_{0} b V \frac{\partial V}{\partial t} \\
& +\alpha+V_{0}(k a)^{2} \sin (k x) .
\end{aligned}
$$

This nonlinear third-order differential equation with derivatives in time has no known analytic solution; however, a special family of solutions represents a propagating wave with a constant velocity. The equation can be integrated to a final second-order nonlinear equation:

$$
\begin{aligned}
& R_{2} C_{0} v a^{2} V^{\prime \prime}+\left(L C_{0} v^{2}-a^{2}\right) V^{\prime}-v R_{1} C_{0} V+R_{1} C_{0} b v V^{2} \\
& =V_{0}(k a)^{2} \cos (k \xi) / k+\alpha \xi
\end{aligned}
$$


Fig. 5 Solutions of Eq. 2 in phase space. In the egg-shaped region, trajectories circulate clockwise, eventually moving far enough outwards to escape to infinity

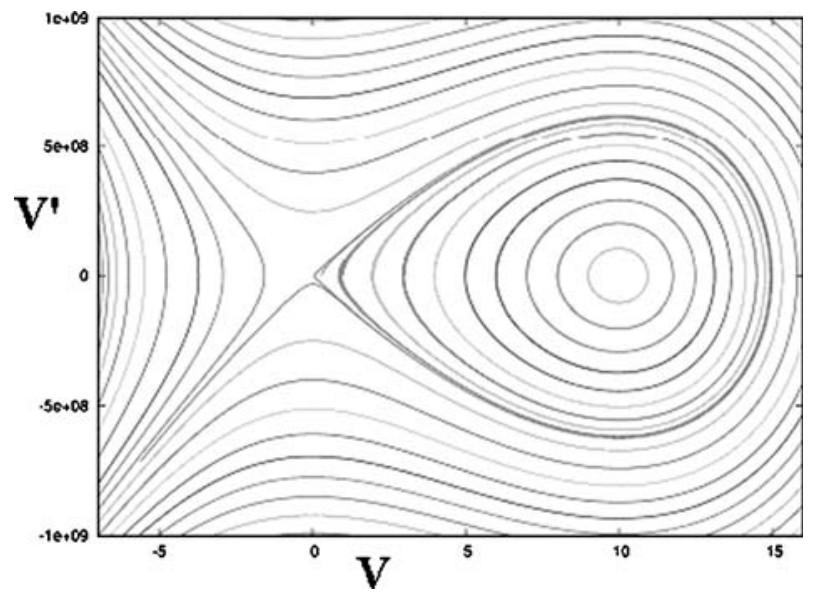

where $\xi$ is a traveling wave coordinate, i.e., $x-v t$. Numerical analysis of the equations' properties was obtained for realistic values of the electric circuit components, as follows: $C_{0}$ is approximately equal to $6.610^{-4} \mathrm{pF}, R_{2}$ equals approximately $1.2 \mathrm{M} \Omega$, and $L$ is approximately $3 \mathrm{pH}$.

Plotting some of the solutions for various initial conditions in phase space produces a phase portrait shown in Fig. 5 where the first-order derivative $V^{\prime}$ is depicted vs. the potential $V$.

The obtained solutions represent oscillations with increasing amplitude. The mathematical similarity between this equation and the one obtained for the actin case [53] implies the possibility of a solitary wave solution.

\section{Dendritic cytoskeleton information processing model}

The Turing machine has provided the conceptual framework for many models of activity in large neuron assemblies. In these models, individual tasks have to be preprogrammed; in other words, an algorithm has to be written to instruct the processor how to manipulate the data at multiple steps until the computational process is finally halted. Each computation in these models is entirely separate from the last, since the input available at the beginning of the operation is lost by the time a subsequent computation for another input commences. Artificial neural network (ANN) paradigms have been proposed to overcome some of the difficulties in solving complex problems inherent in pattern recognition, temporal sequences processing, and similar tasks. ANN models rely on massively interconnected parallel networks of simple units, which are analogous to neurons, and on a learning algorithm to train, or adapt, the parameters of the model. Depending on the type of ANN, the parameters of the model may be the strength of connections between the model's units (i.e., neurons), the number of units required to perform the task, the connectivity in the network, or the fine structure of the artificial neuron [56, 57, 76-79]. ANN models have been very successful at solving problems that are intrinsically "static," where the task is time-independent. In order to deal with temporal or time-dependent problems, some ANN models have been extended to include timing parameters, and alternative models have been proposed $[58,80]$. Despite 
these adaptations, a remaining problem with these models is most evident with continuous streams of data. Models incorporating attractor dynamics present difficulties insofar as huge numbers of attractors are required to represent the information and long time intervals are required for the dynamic system to converge to a solution attractor. Additionally, the aforementioned models lack a true memory of recent inputs and are therefore unable to process the current information within the context of the recently observed data. As such, these models are inadequate and inappropriate tools with which to study real neurons, in particular the highly dynamic behavior observed during synaptic activation and with neural plasticity [59, 60, 81, 82]. While synapses in ANN vary slowly during the learning process, these synapses are assumed to be static after the learning phase is over. This is inconsistent with activity patterns in real neural assemblies and behavior at actual synapses, which in both cases is highly dynamic and activity-dependent. The ANN model depicts the neuron simplistically, which is unlikely to be accurate in a number of crucial ways.

An alternate new concept for real-time neural computation of temporal processing has been proposed recently to explain the existence and function of microcircuits in the brain, in particular in the cortex [84]. In this model, brain-wide neural assemblies, as well as microcircuits, are highly generic, meaning that they are not task-dependent, their dynamics change continuously, and they do not seem to converge to a particular attractor. For example, the computation and its output never converge to a particular dynamic state (i.e., input information arrives continuously, not in one batch). This alternate network concept is based on a non-specific, high-dimensional dynamical system, serving as a source of trajectories, called a "liquid state machine" (LSM) [85, 86]. A very similar idea was proposed independently by another group [87] under the name of "echo state networks." The basic structure of an LSM is composed of an excitable medium (i.e., a "liquid"), with the output function mapping the current liquid state, as shown in Fig. 6.

The liquid module must be sufficiently complex and dynamic to guarantee universal computational power and to ensure that different input excitations will lead to separate trajectories in the internal states of the machine. These requirements of the model have been rigorously proven [88]. Accordingly, the output function, $f^{M}$, is trained on a specific task. Examples of a "liquid" include a network of spiking neurons and a recurrent neural network. The output function, or readout, for these neural applications has been implemented by simple perceptrons, threshold functions, or linear regression functions. It is clear that a simple readout function restricts the ability of the whole system to capture complex nonlinear dependencies.

Fig. 6 The structure of a liquid state machine $(L S M)$. Continuous stream of input data $i(t)$ is injected to the liquid module $\mathrm{L}^{\mathrm{M}}$ which evolves its internal state, $x^{M}(t)$; the internal state is transformed by the readout module, $f^{M}(t)$, to generate the output stream, $o(t)$

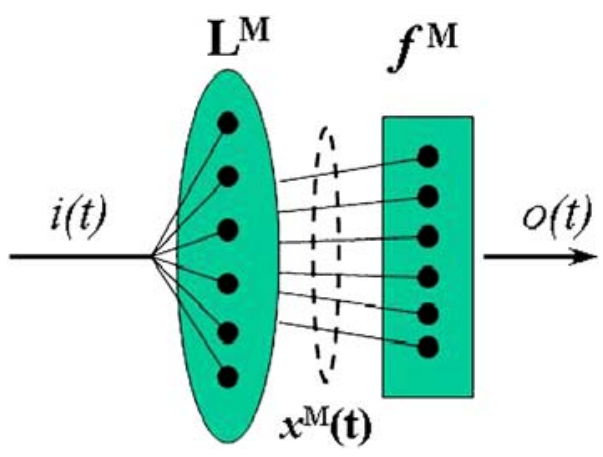


Building on the LSM model and its applications, and taking into account the experimental and theoretical results regarding nonlinear wave propagation along MTs and actin filaments, it can be further hypothesized that the cytoskeletal biopolymers responsible for ionic wave propagation throughout the neuron behave as a sub-neural LSM. The core concept is that the cytoskeleton matrix interacts with and regulates neural membrane components such as ion channels, receptors for transmitters, adaptor proteins, and scaffolding proteins. Figure 7 illustrates the cytoskeleton at the neural cell level, whereas Fig. 8 depicts a portion of the dendritic shaft where microtubules (MTs) are interconnected by MAP2. Connections between MTs and actin filaments, which are integral to the model, are shown as well. The analogy to a liquid state machine is based on the following observations:

- A cross-section through a typical-sized dendrite contains 100 MTs [89].

- MTs are highly interconnected by MAP2 creating an intraneuronal network of nanowires.

- The input/output connections to the MT network are provided by actin filaments.

- Each of the network's elements (e.g., actin filaments and microtubules) behaves as a nonlinear electrical component $[53,75,83]$.

According to this hypothesis, a mechanism exists in which either actin filaments or MTs directly regulate ion channels or receptors and subsequently affect synaptic strength. This endows the cytoskeletal matrix with the capacity to control the electrical response of the neuron at large. Accordingly, MTs receive electric signals from synapses and/or ion channels via actin filaments connected to MTs by MAP2 [91] or via direct MT connections to PSD proteins by molecules such as CRIPT [92]. In response to these inputs, the MT matrix may act as a high dimensional dynamic system, or as a liquid module, where the main degrees of freedom are related to the electric flow along each MT. The current state of the system continues to evolve as new input signals arrive. A previously suggested sequence

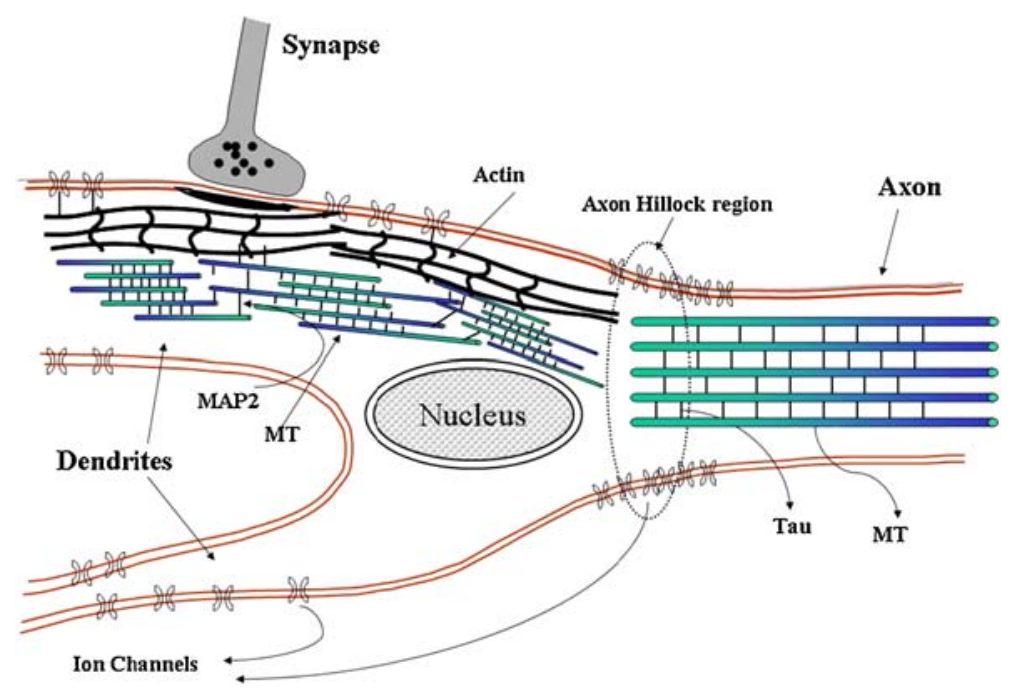

Fig. 7 Each neuron contains microtubules (MTS) interconnected by MAP2 (in the dendrite) and MAP-tau (in the axon). Connections between MTs and actin filaments are shown as well as actin linkage to the membrane. This figure has been adapted from $[93,94]$ 
Fig. 8 Cut-away view of the dendritic shaft where microtubules (MTs) are interconnected by MAP2. Connections between MTs and actin filaments are shown as well. Actin bundles bind to the postsynaptic density (PSD). On the upper left-hand side, a spiny synapse is shown where actin bundles enter the spine neck and bind to the PSD. This figure has been adapted from $[93,94]$

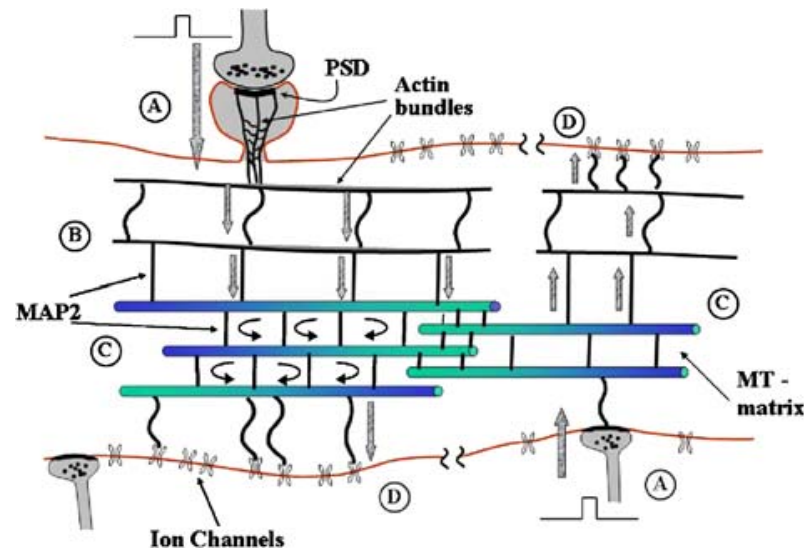

of events [93] is outlined in Fig. 8 as follows: electrical signals arrive at the PSD as a consequence of traditional synaptic transmission; these electrical signals in turn transmit ionic waves along actin filaments (Fig. 8a); next, these electrical signals propagate in the form of ionic waves through actin filaments to the MT matrix (Fig. 8b); and finally, the MT network operates as a high dimensional state machine, evolving these input states by dynamically changing the flow associated with individual MTs (Fig. 8c) and/or by supporting nonlinear wave collisions. The computed output from the MT matrix is the state of the system at a time ' $T$ ' that is being transmitted by actin filaments to remote ion channels. This output function is presumably responsible for regulating the temporal gating state of voltage-sensitive channels (Fig. 8d). One particularly interesting case is when electrical signals transmitted along the cytoskeleton regulate the membrane potential at the axon hillock by changing the distribution and topology of open versus closed voltagegated channels. This represents a unique opportunity for cytoskeletal signaling to regulate neuronal firing.

\section{Conclusions}

There are specific biophysical properties of actin filaments and microtubules enabling them to conduct ionic currents and participate in processing information. The biophysical properties of these filaments relevant to the conduction of ionic current include highly charged surfaces, a condensation of counterions on the filament surface, and a complex nonlinear physical structure. Possible roles for cable-like, conductive filaments in neurons include intracellular information processing, regulating synaptic input, modulating neural firing, regulation of developmental plasticity, and mediation of transport. Operating as a widespread interconnected matrix, cytoskeletal proteins form a complex network capable of emergent information processing, taking into account activities throughout the neuron. This cytoskeletal matrix critically intervenes between inputs to and outputs from neurons by receiving information from the neuronal membrane and its intrinsic components (e.g., ion channels, scaffolding proteins, and adaptor proteins), especially at sites of synaptic contacts and spines, and in turn affecting the output of the neuron. An information processing model based on cytoskeletal networks has been outlined in this paper. This model may underlie certain types of learning and memory, as well as have applicability to learning and memory function in general. 
A specific concept developed in this paper is that cytoskeletal structures may behave as a liquid state machine. This proposal provides a means for real-time computation without the need for stable attractors. Moreover, the output in this model is relatively insensitive to small variations in either the MT matrix or the input stream. Nonetheless, recent perturbations do have a long-term effect on the dynamic trajectories such that there is a memory effect inherent to this system. According to the model, the temporal behavior of ion channel function could be regulated by the output from the matrix, which may be linear functions that converge at or near ion channels. This model can also be applied to synaptic strengthening, LTP, and memory enhancement. In these cases, the output function reflects an effect of the MT matrix on synaptic channel function such that the desired state of the channel appears in a higher open probability. Hebbian-based responses can be modeled as more frequent activity of certain sub-domains of the MT matrix, whose output states give rise to higher/lower densities of actin filaments connecting to corresponding channels.

Acknowledgements This research was supported by funding from the Natural Sciences and Engineering Research Council of Canada (NSERC), the Allard Foundation, the Alberta Cancer Foundation and Alberta's Advanced Education and Technology awarded to JAT. The authors wish to thank Dr. T. Luchko for his help in generating some artwork for this article.

Open Access This article is distributed under the terms of the Creative Commons Attribution Noncommercial License which permits any noncommercial use, distribution, and reproduction in any medium, provided the original author(s) and source are credited.

\section{References}

1. Lynch, G., Rex, C.S., Chen, L.Y., Gall, C.M.: The substrates of memory: defects, treatments, and enhancement. Eur. J. Pharmacol. 585(1), 2-13 (2008). doi:10.1016/j.ejphar.2007.11.082

2. Prager-Khoutorsky, M., Spira, M.E.: Neurite retraction and regrowth regulated by membrane retrieval, membrane supply, and actin dynamics. Brain Res. 1251, 65-79 (2009)

3. Yamaguchi, S., Fujii-Taira, I., Murakami, A., Hirose, N., Aoki, N., Izawa, E., Fujimoto, Y., Takano, T., Matsushima, T., Homma, K.J.: Up-regulation of microtubule-associated protein 2 accompanying the filial imprinting of domestic chicks (Gallus gallus domesticus). Brain Res. Bull. 76(3), 282-288 (2008). doi:10.1016/j.brainresbull.2008.02.010

4. Ma, J.: A hybrid neural network of addressable and content-addressable memory. Int. J. Neural Syst. 13(3), 205-213 (2003)

5. Swindale, N.V.: Feedback decoding of spatially structured population activity in cortical maps. Neural Comput. 20(1), 176-204 (2008)

6. O’Connell, C., O’Malley, A., Regan, C.M.: Transient, learning-induced ultrastructural change in spatially-clustered dentate granule cells of the adult rat hippocampus. Neuroscience 76(1), 55-62 (1997)

7. Nakayama, T., Sawada, T.: Involvement of microtubule integrity in memory impairment caused by colchicine. Pharmacol. Biochem. Behav. 71(1-2), 119-138 (2002)

8. Bensimon, G., Chermat, R.: Microtubule disruption and cognitive defects: effect of colchicine on learning behavior in rats. Pharmacol. Biochem. Behav. 38(1), 141-145 (1991)

9. Di Patre, P.L., Oh, J.D., Simmons, J.M., Butcher, L.L.: Intrafimbrial colchicine produces transient impairment of radial-arm maze performance correlated with morphologic abnormalities of septohippocampal neurons expressing cholinergic markers and nerve growth factor receptor. Brain Res. 523(2), 316-320 (1990)

10. Mileusnic, R., Lancashire, C.L., Rose, S.P.: Recalling an aversive experience by day-old chicks is not dependent on somatic protein synthesis. Learn. Mem. 12(6), 615-619 (2005)

11. Cavallaro, S., D’Agata, V., Manickam, P., Dufour, F., Alkon, D.L.: Memory-specific temporal profiles of gene expression in the hippocampus. Proc. Natl. Acad. Sci. U. S. A. 99(25), 16279-16284 (2002)

12. Nelson, T.J., Backlund, P.S., Jr., Alkon, D.L.: Hippocampal protein-protein interactions in spatial memory. Hippocampus 14(1), 46-57 (2004). doi:10.1002/hipo.10152 
13. Mori, N., Morii, H.: SCG10-related neuronal growth-associated proteins in neural development, plasticity, degeneration, and aging. J. Neurosci. Res. 70(3), 264-273 (2002)

14. Bianchi, M., Fone, K.F., Azmi, N., Heidbreder, C.A., Hagan, J.J., Marsden, C.A.: Isolation rearing induces recognition memory deficits accompanied by cytoskeletal alterations in rat hippocampus. Eur. J. Neurosci. 24(10), 2894-2902 (2006)

15. Woolf, N.J.: A structural basis for memory storage in mammals. Prog. Neurobiol. 55(1), 59-77 (1998)

16. Woolf, N.J., Zinnerman, M.D., Johnson, G.V.: Hippocampal microtubule-associated protein-2 alterations with contextual memory. Brain Res. 821(1), 241-249 (1999)

17. Woolf, N.J., Young, S.L., Johnson, G.V., Fanselow, M.S.: Pavlovian conditioning alters cortical microtubule-associated protein-2. NeuroReport 5(9), 1045-1048 (1994)

18. Woolf, N.J.: Cholinoceptive cells in rat cerebral cortex: somatodendritic immunoreactivity for muscarinic receptor and cytoskeletal proteins. J. Chem. Neuroanat. 6(6), 375-390 (1993)

19. Van der Zee, E.A., Douma, B.R., Bohus, B., Luiten, P.G.: Passive avoidance training induces enhanced levels of immunoreactivity for muscarinic acetylcholine receptor and coexpressed PKC gamma and MAP-2 in rat cortical neurons. Cereb. Cortex 4(4), 376-390 (1994)

20. Liu, H.X., Zhang, J.J., Zheng, P., Zhang, Y.: Altered expression of MAP-2, GAP-43, and synaptophysin in the hippocampus of rats with chronic cerebral hypoperfusion correlates with cognitive impairment. Brain Res. Mol. Brain Res. 139(1), 169-177 (2005)

21. Shimada, A., Tsuzuki, M., Keino, H., Satoh, M., Chiba, Y., Saitoh, Y., Hosokawa, M.: Apical vulnerability to dendritic retraction in prefrontal neurones of ageing SAMP10 mouse: a model of cerebral degeneration. Neuropathol. Appl. Neurobiol. 32(1), 1-14 (2006)

22. Khuchua, Z., Wozniak, D.F., Bardgett, M.E., Yue, Z., McDonald, M., Boero, J., Hartman, R.E., Sims, H., Strauss, A.W.: Deletion of the N-terminus of murine map2 by gene targeting disrupts hippocampal ca1 neuron architecture and alters contextual memory. Neuroscience 119(1), 101-111 (2003). doi:10.1016/S0306-4522(03)00094-0

23. Mershin, A., Pavlopoulos, E., Fitch, O., Braden, B.C., Nanopoulos, D.V., Skoulakis, E.M.: Learning and memory deficits upon TAU accumulation in Drosophila mushroom body neurons. Learn. Mem. 11(3), 277-287 (2004)

24. Pennanen, L., Wolfer, D.P., Nitsch, R.M., Götz, J.: Impaired spatial reference memory and increased exploratory behavior in P301L tau transgenic mice. Genes Brain Behav. 5(5), 369-379 (2006)

25. Boekhoorn, K., Terwel, D., Biemans, B., Borghgraef, P., Wiegert, O., Ramakers, G.J., de Vos, K., Krugers, H., Tomiyama, T., Mori, H., Joels, M., van Leuven, F., Lucassen, P.J.: Improved long-term potentiation and memory in young tau-P301L transgenic mice before onset of hyperphosphorylation and tauopathy. J. Neurosci. 26(13), 3514-3523 (2006)

26. Bianchi, M., Heidbreder, C., Crespi, F.: Cytoskeletal changes in the hippocampus following restraint stress: role of serotonin and microtubules. Synapse 49(3), 188-194 (2003). doi:10.1002/syn.10230

27. Reinés, A., Cereseto, M., Ferrero, A., Bonavita, C., Wikinski, S.: Neuronal cytoskeletal alterations in an experimental model of depression. Neuroscience 129(3), 529-538 (2004)

28. Guest, P.C., Knowles, M.R., Molon-Noblot, S., Salim, K., Smith, D., Murray, F., Laroque, P., Hunt, S.P., De Felipe, C., Rupniak, N.M., McAllister, G.: Mechanisms of action of the antidepressants fluoxetine and the substance $\mathrm{P}$ antagonist L-000760735 are associated with altered neurofilaments and synaptic remodeling. Brain Res. 1002(1-2), 1-10 (2004)

29. Fischer, A., Sananbenesi, F., Schrick, C., Spiess, J., Radulovic, J.: Distinct roles of hippocampal de novo protein synthesis and actin rearrangement in extinction of contextual fear. J. Neurosci. 24(8), 1962-1966 (2004)

30. Yuste, R., Bonhoeffer, T.: Morphological changes in dendritic spines associated with long-term synaptic plasticity. Annu. Rev. Neurosci. 24, 1071-1089 (2001). doi:10.1146/annurev. neuro.24.1.1071

31. Roberts, L.A., Large, C.H., Higgins, M.J., Stone, T.W., O’Shaughnessy, C.T., Morris, B.J.: Increased expression of dendritic mRNA following the induction of long-term potentiation. Brain Res. Mol. Brain Res. 56(1-2), 38-44 (1998)

32. Fukunaga, K., Muller, D., Miyamoto, E.: CaM kinase II in long-term potentiation. Neurochem. Int. 28(4), 343-358 (1996)

33. Zervas, M., Opitz, T., Edelmann, W., Wainer, B., Kucherlapati, R., Stanton, P.K.: Impaired hippocampal long-term potentiation in microtubule-associated protein 1B-deficient mice. J. Neurosci. Res. 82(1), 8392 (2005)

34. Golomb, D., Hansel, D.: The number of synaptic inputs and the synchrony of large, sparse neuronal networks. Neural Comput. 12(5), 1095-1139 (2000)

35. Korol, D.L., Gold, P.E.: Epinephrine converts long-term potentiation from transient to durable form in awake rats. Hippocampus 18(1), 81-91 (2008). doi:10.1002/hipo.20372 
36. Rioult-Pedotti, M.S., Donoghue, J.P., Dunaevsky, A.: Plasticity of the synaptic modification range. J. Neurophysiol. 98(6), 3688-3695 (2007)

37. Woolf, N.J.: Microtubules in the cerebral cortex: role in memory and consciousness. In: Tuszynski, J. (ed.) The Emerging Physics of Consciousness. Springer, Berlin (2006)

38. Rioult-Pedotti, M.S., Friedman, D., Donoghue, J.P.: Learning-induced LTP in neocortex. Science 290(5491), 533-536 (2000). doi:10.1126/science.290.5491.533

39. Monfils, M.H., Teskey, G.C.: Skilled-learning-induced potentiation in rat sensorimotor cortex: a transient form of behavioural long-term potentiation. Neuroscience 125(2), 329-336 (2004). doi:10.1016/j.neuroscience.2004.01.048

40. Rogan, M.T., Stäubli, U.V., LeDoux, J.E.: Fear conditioning induces associative long-term potentiation in the amygdala. Nature 390, 604-607 (1997). doi:10.1038/37601

41. Withers, G.S., Greenough, W.T.: Reach training selectively alters dendritic branching in subpopulations of layer II-III pyramids in rat motor-somatosensory forelimb cortex. Neuropsychologia 27, 61-69 (1989). doi:10.1016/0028-3932(89)90090-0

42. Escobar, G., Fares, T., Stepanyants, A.: Structural plasticity of circuits in cortical neuropil. J. Neurosci. 28(34), 8477-8488 (2008). doi:10.1523/JNEUROSCI.2046-08.2008

43. Benavides-Piccione, R., Ballesteros-Yáñez, I., DeFelipe, J., Yuste, R.: Cortical area and species differences in dendritic spine morphology. J. Neurocytol. 31(3-5), 337-346 (2002). doi:10.1023/A:1024134312173

44. Bender, V.A., Bender, K.J., Brasier, D.J., Feldman, D.E.: Two coincidence detectors for spike timingdependent plasticity in somatosensory cortex. J. Neurosci. 26(16), 4166-4177 (2006)

45. Tsukada, M., Pan, X.: The spatiotemporal learning rule and its efficiency in separating spatiotemporal patterns. Biol. Cybern. 92(2), 139-146 (2005)

46. Tsukada, M., Aihara, T., Saito, H.A., Kato, H.: Hippocampal LTP depends on spatial and temporal correlation of inputs. Neural Netw. 9(8), 1357-1365 (1996)

47. Goldman, J.E.: Immunocytochemical studies of actin localization in the central nervous system. J. Neurosci. 3(10), 1952-1962 (1983)

48. Honkura, N., Matsuzaki, M., Noguchi, J., Ellis-Davies, G.C., Kasai, H.: The subspine organization of actin fibers regulates the structure and plasticity of dendritic spines. Neuron 57(5), 719-729 (2008)

49. Star, E.N., Kwiatkowski, D.J., Murthy, V.N.: Rapid turnover of actin in dendritic spines and its regulation by activity. Nat. Neurosci. 5(3), 239-246 (2002)

50. Matus, A.: Actin-based plasticity in dendritic spines. Science 290(5492), 754-758 (2000)

51. Gomez, L.L., Alam, S., Smith, K.E., Horne, E., Dell'Acqua, M.L.: Regulation of A-kinase anchoring protein 79/150-cAMP-dependent protein kinase postsynaptic targeting by NMDA receptor activation of calcineurin and remodeling of dendritic actin. J. Neurosci. 22(16), 7027-7044 (2002)

52. Cantiello, H.F., Patenaude, C., Zaner, K.: Osmotically induced electrical signals from actin filaments. Biophys. J. 59(6), 1284-1289 (1991)

53. Tuszyński, J.A., Portet, S., Dixon, J.M., Luxford, C., Cantiello, H.F.: Ionic wave propagation along actin filaments. Biophys. J. 86(4), 1890-1903 (2004)

54. Otterbein, L.R., Graceffa, P., Dominguez, R.: The crystal structure of uncomplexed actin in the ADP state. Science 293(5530), 708-711 (2001)

55. Rould, M.A., Wan, Q., Joel, P.B., Lowey, S., Trybus, K.M.: Crystal structures of expressed nonpolymerizable monomeric actin in the ADP and ATP states. J. Biol. Chem. 281(42), 31909-31919 (2006)

56. Trizac, E., Téllez, G.: Onsager-Manning-Oosawa condensation phenomenon and the effect of salt. Phys. Rev. Lett. 96(3), 038302 (2006)

57. Angelini, T.E., Liang, H., Wriggers, W., Wong, G.C.: Like-charge attraction between polyelectrolytes Induced by counterion charge density waves. Proc. Natl. Acad. Sci. U. S. A. 100(15), 8634-8637 (2003)

58. Lin, E.C., Cantiello, H.F.: A novel method to study the electrodynamic behavior of actin filaments. Evidence for cable-like properties of actin. Biophys. J. 65(4), 1371-1378 (1993)

59. Arsenault, M.E., Zhao, H., Purohit, P.K., Goldman, Y.E., Bau, H.H.: Confinement and manipulation of actin filaments by electric fields. Biophys. J. 93(8), L42-44 (2007)

60. Borejdo, J., Ortega, H.: Electrophoresis and orientation of F-actin in agarose gels. Biophys. J. 56(2), 285-293 (1989)

61. Kobayasi, S., Asai, H., Oosawa, F.: Electric birefringence of actin. Biochim. Biophys. Acta 88, 528-540 (1964)

62. Meggs, W.J.: Electric fields determine the spatial organization of microtubules and actin filaments. Med. Hypotheses 26(3), 165-170 (1988)

63. Tuszyński, J.A., Luchko, T., Portet, S., Dixon, J.M.: Anisotropic elastic properties of microtubules. Eur. Phys. J. E Soft Matter 17(1), 29-35 (2005) 
64. Sept, D., Baker, N.A., McCammon, J.A.: The physical basis of microtubule structure and stability. Protein Sci. 12(10), 2257-2261 (2003)

65. Diaz, J.F., Andreu, J.M., Diakun, G., Towns-Andrews, E., Bordas, J.: Structural intermediates in the assembly of taxoid-induced microtubules and GDP-tubulin double rings: time-resolved X-ray scattering. Biophys. J. 70(5), 2408-2420 (1996)

66. Krebs, A., Goldie, K.N., Hoenger, A.: Structural rearrangements in tubulin following microtubule formation. EMBO Rep. 6(3), 227-232 (2005)

67. Baker, N.A., Sept, D., Joseph, S., Holst, M.J., McCammon, J.A.: Electrostatics of nanosystems: application to microtubules and the ribosome. Proc. Natl. Acad. Sci. U. S. A. 98(18), 10037-10041 (2001)

68. Minoura, I., Muto, E.: Dielectric measurement of individual microtubules using the electroorientation method. Biophys. J. 90(10), 3739-3748 (2006)

69. Tuszynski, J., Hameroff, S., Sataric, M.V., Trpisova, B., Nip, M.L.A.: Ferroelectric behavior in microtubule dipole lattices: implications for information processing, signaling and assembly/disassembly. J. Theor. Biol. 174, 371 (1995). doi:10.1006/jtbi.1995.0105

70. Satarić, M.V., Tuszyński, J.A.: Relationship between the nonlinear ferroelectric and liquid crystal models for microtubules. Phys. Rev. E 67(1 Pt 1), 011901 (2003)

71. Chen, Y., Qiu, X.J., Dong, X.L.: Pseudo-spin model for the microtubule wall in external field. Biosystems 82(2), 127-136 (2005)

72. Sataric, M.V., Tuszynski, J.A., Zakula, R.B.: Kinklike excitations as an energy-transfer mechanism in microtubules. Phys. Rev. E 48(1), 589-597 (1993)

73. Mershin, A., Kolomenski, A.A., Schuessler, H.A., Nanopoulos, D.V.: Tubulin dipole moment, dielectric constant and quantum behavior: computer simulations, experimental results and suggestions. Biosystems 77(1-3), 73-85 (2004)

74. Priel, A., Ramos, A.J., Tuszynski, J.A., Cantiello, H.F.: A biopolymer transistor: electrical amplification by microtubules. Biophys. J. 90(12), 4639-4643 (2006)

75. Priel, A., Tuszynski, J.A.: A nonlinear cable-like model of amplified ionic wave propagation along microtubules. Eur. Phys. Lett. 83, (68004) (2008)

76. Shikinaka, K., Kwon, H., Kakugo, A., Furukawa, H., Osada, Y., Gong, J.P., Aoyama, Y., Nishioka, H., Jinnai, H., Okajima, T.: Observation of the three-dimensional structure of actin bundles formed with polycations. Biomacromolecules 9(2), 537-542 (2008)

77. Bishop, C.M.: Pattern Recognition and Machine Learning. Springer, New York (2006)

78. Duda, Hart and Stork, Pattern Classification, Wiley \& Sons (2000)

79. Haykin, S.: Neural Networks and Learning Machines. Third Edition. Prentice Hall (2008)

80. Mandic, D., Chambers, J.: Recurrent Neural Networks for Prediction: Learning Algorithms, Architectures and Stability. Wiley (2001)

81. Abbott, L.F., Regehr, W.G.: Synaptic computation. Nature 431, 796-803 (2004)

82. Natschlager, T., Maass, W., Zador, A.: Efficient temporal processing with dynamic synapses. Network 12, 75-87 (2001)

83. Priel, A., Tuszynski, J.A., Woolf, N.J.: Transitions in microtubule C-termini conformations as a possible dendritic signaling phenomenon. Eur Biophys J. 35(1), 40-52 (2005)

84. Kalisman, N., Silberberg, G., Markram, H.: The neocortical microcircuit as a tabula rasa. Proc. Natl. Acad. Sci. U. S. A. 102(3), 880-885 (2005). doi:10.1073/pnas.0407088102

85. Maass, W., Natschläger, T., Markram, H.: Real-time computing without stable states: a new framework for neural computation based on perturbations. Neural Comput. 14(11), 2531-2560 (2002). doi:10.1162/089976602760407955

86. Maass, W., Natschläger, T., Markram, H.: A model for real-time computation in generic neural microcircuits. In: Becker, S., Thrun, S., Obermayer, K. (eds.) Proceedings of NIPS 2002, Advances in Neural Information Processing Systems, vol. 15, pp. 229-236. MIT Press, Cambridge, MA (2003)

87. Jaeger, H.: The "echo state" approach to analysing and training recurrent neural networks. GMD report 148, GMD - German National Research Institute for Computer Science (2001)

88. Maass, W., Markram, H.: On the computational power of recurrent circuits of spiking neurons. J. Comput. Syst. Sci. 69(4), 593-616 (2004). doi:10.1016/j.jcss.2004.04.001

89. Leclerc, N., Baas, P.W., Garner, C.C., Kosik, K.S.: Juvenile and mature MAP2 isoforms induce distinct patterns of process outgrowth. Mol. Biol. Cell 7(3), 443-455 (1996)

90. Watson, G.S., Cahill, C., Blach, J., Myhra, S., Alexeeva, Y., Ivanova, E.P., Nicolau, D.V.: Actin nanotracks for hybrid nanodevices based on linear protein molecular motors. In Advanced microsystemsintegration with nanotechnology and biomaterials. In: Borenstein, J.T., Grodzinski, P., Lee, L.P., Liu, J., Wang, Z. (eds.) Nanoengineered Assemblies and Advanced Micro/Nanosystems. MRS, San Francisco, O2.4 (2004) 
91. Rodriguez, O.C., Schaefer, A.W., Mandato, C.A., Forscher, P., Bement, W.M., Waterman-Storer, C.M.: Conserved microtubule-actin interactions in cell movement and morphogenesis. Nat. Cell Biol. 5, 599609 (2003). doi:10.1038/ncb0703-599

92. Passafaro, M., Sala, C., Niethammer, M., Sheng, M.: Microtubule binding by CRIPT and its potential role in the synaptic clustering of PSD-95. Nat. Neurosci. 2, 1063-1069 (1999). doi:10.1038/15990

93. Priel, A., Tuszynski, J.A., Cantiello, H.F.: Electrodynamic signaling by the dendritic cytoskeleton: toward an intracellular information processing model. Electromagnetic Biology Medicine 24(3), 221231 (2005). doi:10.1080/15368370500379590

94. Priel, A., Tuszynski, J.A., Cantiello, H.: Ionic waves propagation along the dendritic cytoskeleton as a signaling mechanism. In: Bittar, E., Khurana, S. (eds.) Molecular Biology of the Cell, vol. 37. Elsevier, Amsterdam (2006) 